Article

\title{
Herramienta de simulación para el análisis de flujo óptimo clásico utilizando multiplicadores de Lagrange ${ }^{*}$
}

\author{
Diego Anzola ${ }^{1}$, Julio Castro ${ }^{1}$, Diego Giral ${ }^{1} \bowtie(\mathbb{D}$ \\ 1 Facultad Tecnológica, Universidad Distrital Francisco José de Caldas, Bogotá D.C 11021, Colombia.

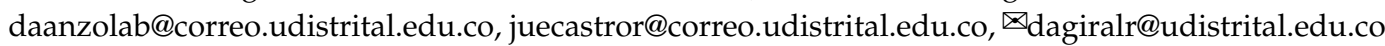

Received: 8 february 2020; Accepted: 18 may 2020; Published: 30 july 2021

\begin{abstract}
The optimal flow analysis is a complex and challenging problem because of its non-linear characteristics. It is difficult to determine the respective flow of active power due to the inclusion of power restrictions and models of the transmission lines. Lagrange multipliers are a classical optimization method that allows solving the economic flow of multiple variables subject to various limits. This article presents a simulation tool called SOPF (Software Optimal Power Flow) developed in Guide-Matlab. This tool analyzes the classical optimal flow problem of a power system with leaks and energetic power limitations. This simulator is an academic support tool for students, professors, and people interested in applying optimization algorithms for economic electrical power systems. The software not only determines the flow of the power of each generator, the costs of the generated flow power, the contribution of each machine, the incremental costs, and the leaks according to the power balance. Finally, the results are presented through two case studies: classic optimal flow with losses and without active power restrictions and classical optimal flow with leaks and brisk power restrictions. For both cases, errors of less than $1 \%$ are obtained.
\end{abstract}

Keywords: Economic dispatch; Optimal power flow; Lagrange multipliers; Nonlinear optimization.

Resumen: El análisis del flujo óptimo es un problema complejo y desafiante por sus características no lineales. La inclusión de restricciones de potencia y los modelos de las líneas de transmisión hacen complejo determinar el respectivo despacho. Los multiplicadores de Lagrange son un método de optimización clásico que permite solucionar problemas de despacho económico de múltiples variables sujetas con diversas restricciones. Este articulo presenta el desarrollo de una herramienta de simulación denominada SOPF (Software Optimal Power Flow), desarrollada en Guide-Matlab y que permite analizar el problema de flujo óptimo clásico de un sistema de potencia con pérdidas y con restricciones de potencia activa, el simulador desarrollado es un herramienta académica de apoyo para los estudiantes, profesores y personas interesadas en la aplicación de algoritmos de optimización para la operación económica de sistemas eléctricos de potencia. Como métricas, el simulador determina el despacho de la potencia activa de cada generador, los costos de generación de la potencia despachada, el aporte de cada máquina, los costos incrementales y las pérdidas de acuerdo al balance de potencia. Finalmente, los resultados se presentan a través de dos casos de estudio: flujo óptimo clásico con pérdidas y sin restricciones de potencia activa y flujo óptimo clásico con pérdidas y con restricciones de potencia activa. Para ambos casos, se obtienen errores inferiores al $1 \%$.

\footnotetext{
${ }^{*}$ How to cite this article: Anzola, Diego; Castro, Julio; Giral, Diego. Herramienta de simulación para el análisis de flujo óptimo clásico utilizando multiplicadores de Lagrange. In Transactions on Energy Systems and Engineering Applications, 1(2): 1-17, 2021. DOI: 10.32397/tesea.vol2.n1.1
} 
Palabras claves: Despacho económico; Flujo óptimo de potencia; Multiplicadores de Lagrange; Optimización no lineal.

\section{Introducción}

\subsection{Contexto General}

El objetivo de un sistema de potencia es operar en un punto de equilibrio, donde todas las magnitudes asociadas al sistema estén dentro de rangos establecidos (Grisales-Noreña et al. 2020). El flujo óptimo de potencia conocido por sus siglas en inglés como OPF, es una extensión del problema de despacho económico de carga convencional (Chen et al. 2016; Das 2017). El OPF, se utiliza para determinar la potencia que debe generar cada unidad del sistema de generación para cubrir la demanda al mínimo costo (Alvarez-Bustos et al. 2021).

El OPF es un problema complejo y desafiante por sus características no lineales, busca minimizar los costos de generación a partir de las funciones de costos asociadas a cada planta, dando prioridad a las centrales de generación más económicas, pero asegurando que todas tengan un porcentaje de participación (Ma and Elia 2019). La inclusión de restricciones de potencia y los modelos de las líneas de transmisión hace complejo determinar el respectivo despacho, es por esto que para la solución del OPF se incluyen modelos de solución basados en estructuras clásicas como Lagrange o técnicas de mayor carga computacional como optimización convexa o estrategias metaheurísticas (Hasan et al. 2020).

El análisis del flujo óptimo a través de Lagrange es sencillo de modelar de forma teórica si el caso de estudio no supera cuatro máquinas de generación. Para casos de estudio con un mayor número de puntos de generación los cálculos aumentan y el proceso de solución teórico es ineficiente. En este punto, y teniendo en cuenta que los sistemas de potencia reales se caracterizan por tener varios nodos, se requiere implementar otra tipo de metodología que permita de forma práctica y sencilla encontrar la solución (Pinheiro et al. 2020).

La simulación es una metodología utilizada para el análisis de sistemas de potencia, es útil debido a que permite analizar prototipos a un costo y tiempo relativamente menor que el requerido para experimentar con implementaciones y desarrollos teóricos. Los simuladores proporcionan un entorno virtual con una variedad de características deseables, que permiten modelar sistemas en función de criterios específicos y analizar su rendimiento en diferentes escenarios (Chaudhary et al. 2012).

\subsection{Revisión de literatura}

Respecto a las investigaciones previas, es importante destacar que no se identificaron trabajos asociados al desarrollo de herramientas de simulación que analicen el OPF a través de Lagrange. Los trabajos que se analizan en este documento se enfocan en investigaciones relevantes relacionadas al desarrollo de simuladores para flujo óptimo.

En (Bukhsh et al. 2020) se desarrolla una herramienta de optimización para el análisis de sistemas de potencia (OATS), la herramienta de simulación es de código abierto bajo la Licencia Pública General GNU (GPLv3). El objetivo es el análisis de estado estable de problemas de sistemas de potencia distribuidos. Permite resolver problemas clásicos de estado estacionario como: flujo de carga y OPF. Para el análisis de OPF permite realizar deslastre de carga y lista de contingencias con restricciones de seguridad. Los modelos de optimización matemática están escritos en un lenguaje de modelado algebraico de código abierto, que ofrece una sintaxis simbólica de alto nivel para describir problemas de optimización.

En (Cuffe 2020) se propone nuevas herramientas para predecir y visualizar los cambios plausibles a corto plazo en la carga de las ramas que pueden surgir debido a las fluctuaciones de producción de los generadores renovables. Para predecir los cambios de carga, se presenta una formulación de flujo 
de potencia óptimo lineal que utiliza una función objetivo novedosa para caracterizar la carga máxima a la que una rama podría estar expuesta a corto plazo.

En (Thurner et al. 2018) se presenta Pandapower, herramienta de análisis de sistemas de potencia con licencia BSD basada en Python que tiene como objetivo la automatización del análisis estático, cuasiestático y la optimización de sistemas de potencia balanceados. Pandapower, proporciona flujo de carga, flujo de carga óptimo, estimación de estado, búsquedas de gráficos topológicos y cálculos de cortocircuito de acuerdo con IEC 60909. La implementación en Python hace que Pandapower sea fácil de usar y permite una extensión cómoda con bibliotecas de terceros. Pandapower se ha aplicado con éxito en varios estudios de redes, así como con fines educativos.

\subsection{Contribución y objetivo}

La contribución y el objetivo de este trabajo es el desarrollo de una herramienta de simulación que permita analizar: flujo óptimo clásico de un sistema de potencia sin pérdidas y sin restricciones de potencia activa, flujo óptimo clásico de un sistema de potencia sin pérdidas y con restricciones de potencia activa, flujo óptimo de un sistema de potencia con pérdidas y sin restricciones de potencia activa y flujo óptimo de un sistema de potencia con pérdidas y con restricciones de potencia activa.

La herramienta de simulación se denomina SOPF (Software Optimal Power Flow), fue desarrollada en Guide-Matlab R2018b y se diseñó con el objetivo de permitir al usuario trabajar bajo un ambiente amigable y parametrizable según las pruebas que se requieran realizar. Como métricas, el simulador determina: el despacho de la potencia activa de cada generador, los costos de generación de la potencia despachada y el aporte de cada máquina en términos porcentuales. Adicionalmente, la herramienta cuenta con la posibilidad de mostrar el resultado obtenido por cada iteración, donde se puede visualizar: potencia generada, costos incrementales, costos de generación, pérdidas y el error.

\subsection{Organización del documento}

Este trabajo está organizado y presentado en cuatro secciones. La sección 2 describe la fundamentación teórica. La sección 3 expone la metodología, en esta se presenta la descripción de los módulos del simulador, definiendo la lógica del módulo, las variables de entrada, salida y los respectivos algoritmos. En la sección 4 se presentan los resultados obtenidos para dos casos específicos. La sección final establece las conclusiones generales del trabajo.

\section{Fundamentación Teórica}

El OPF puede ser representado como un problema de optimización no lineal cuya función objetivo se presenta en la ecuación (1) (Montoya 2017).

$$
\min \sum_{i=1}^{n} C_{i}\left(P_{G i}\right)
$$

Las restricciones del problema de optimización se caracterizan de acuerdo al balance de potencia (ecuación (2)) y a las restricciones del despacho máxima y mínimo de potencia activa (ecuación (3)).

$$
\begin{gathered}
\sum_{i=1}^{n} P_{G i}=\sum P_{D}+P_{L}\left(P_{G}, P_{D}\right) \\
P_{G i}^{\min } \leq P_{G i} \leq P_{G i}^{\max }
\end{gathered}
$$




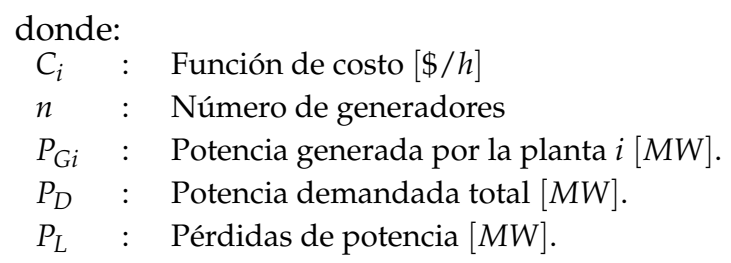

\subsection{Formulación de la función objetivo}

La formulación de la función objetivo se realiza a través del costo de operación de las unidades de generación. Para las centrales térmicas la característica de entrada y salida que permiten analizar el costo de operación se realiza a través de una aproximación cuadrática. La figura (1) presenta el comportamiento del consumo de combustible para una central térmica, donde en el eje de las ordenadas se encuentra la entrada de energía $H[M B t u / h]$ y en el eje de las abscisas la potencia de salida neta en $M W$ (Wood et al. 2013).

La cantidad de energía $H[M B t u / h]$ se multiplica por el costo del combustible que está en unidades

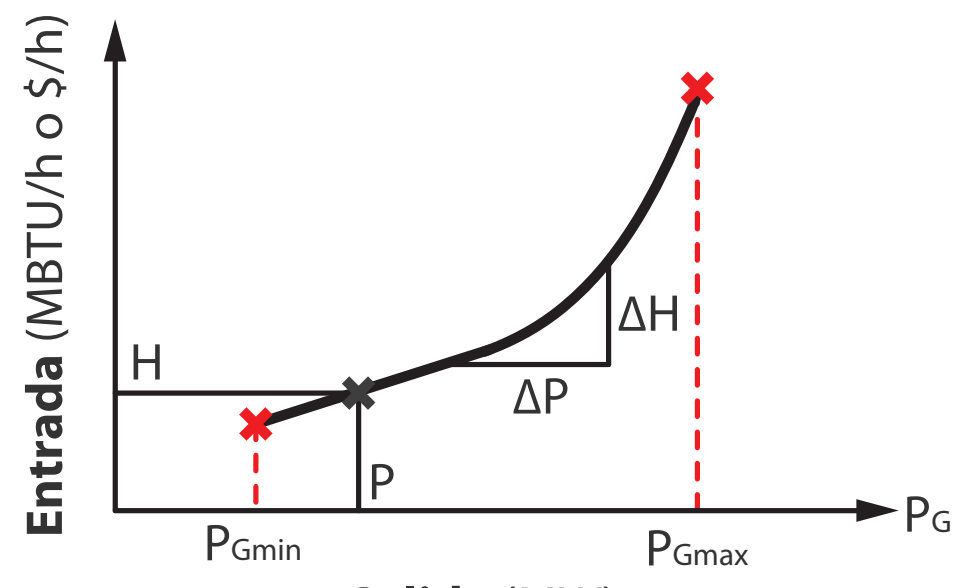

Salida (MW)

Figure 1. Curva característica de entrada-salida de la unidad generadora térmica

de $[\$ / h]$ y se obtiene la función de consumo de combustible (ecuación (4)).

$$
C_{i}\left(P_{G i}\right)=\frac{a_{i}}{2}\left(P_{G i}\right)^{2}+b_{i}\left(P_{G i}\right)+c_{i} \quad\left[\frac{\$}{h}\right]
$$

\subsection{Metodología de solución}

Cuando entra en operación plantas térmicas e hidráulicas, se busca que las plantas hidráulicas generen más potencia dado que sus costos de generación son menores respecto a las plantas térmicas. El método planteado que se presenta en este artículo es válido solo para despachos que involucren plantas térmicas. El método a implementar es el de los multiplicadores de Lagrange dado que permite encontrar la solución cuando existe más de una variable (Ilyas et al. 2020).

El método de los multiplicadores de Lagrange define que para determinar los extremos relativos de una función $f$ de dos variables $x$ y y sujetas a la restricción $g(x, y)=0$, se debe definir una función auxiliar $l$ de las tres variables $x, y$ y $\lambda$ como se muestra en la ecuación (5).

$$
l(x, y, \lambda)=f(x, y)+\lambda g(x, y)
$$


Posteriormente de acuerdo al criterio de primer orden, se igualan a cero las derivadas parciales de la ecuación (5), formando el sistema de ecuaciones (6), (7), (8).

$$
\begin{aligned}
& l_{x}(x, y, \lambda)=0 \\
& l_{y}(x, y, \lambda)=0 \\
& l_{\lambda}(x, y, \lambda)=0
\end{aligned}
$$

Al resolver el sistema de ecuaciones se determina los puntos críticos de $f$, donde estos dos primeros valores obtenidos corresponden a los extremos relativos de $x$ y $y$.

\subsection{Despacho sin pérdidas de potencia activa}

Es un despacho sin pérdidas de potencia activa $\left(P_{L}=0\right)$ y sin límites de generación, se define como OPF clásico sin restricciones. Bajo este escenario se supone que la demanda del sistema es inelástica, por lo tanto, el objetivo de este despacho es maximizar la diferencia entre el beneficio de la demanda y el costo de generación. Para el análisis del OPF clásico sin restricciones, los multiplicadores de Lagrange permiten encontrar el despacho de las máquinas de generación (ecuación (9)).

$$
l(P, \lambda)=\left(\sum_{n=1}^{i} C_{n}\left(P_{G n}\right)\right)+\lambda\left(\sum P_{D}-\sum_{n=1}^{i} P_{G n}\right)
$$

De acuerdo a la condición de primer orden, se obtiene la ecuación (10) y la ecuación (11). Donde la ecuación (10) permite determinar la potencia que debe generar cada máquina conectada al sistema y la ecuación (11) representa el balance de potencia.

$$
\begin{gathered}
\frac{\partial l_{i}}{\partial P_{G i}}=\frac{\partial C_{i}}{P_{G i}}-\lambda_{i}=0 \\
\frac{\partial l}{\partial \lambda}=-\sum_{n=1}^{i} P_{G n}+\sum P_{D}=0
\end{gathered}
$$

2.3.1. Condiciones de primer orden $\frac{\partial l_{i}}{\partial P_{G i}}$

La condición de primer orden (ecuación (9)) establece la potencia activa $P_{G i}$ que debe despachar cada máquina, esta condición incluye el costo incremental $(\lambda)$, factor que se presenta en la ecuación (12). El costo incremental es el costo adicional en dólares por hora para incrementar la salida en un 1 MW. Cuando se tienen las funciones de costos en forma cuadrática, como en la ecuación (4), el costo incremental se representa a través de la ecuación (12).

$$
\lambda_{i}=\frac{\partial C_{i}\left(P_{G i}\right)}{\partial P_{G i}}=a_{i}\left(P_{G i}\right)+b_{i}
$$

Despejando $P g_{i}$ de la ecuación (12) en términos de $a_{i}, b_{i}$ y $\lambda_{i}$ se obtiene la ecuación (13).

$$
P_{G i}=\frac{\lambda_{i}-b_{i}}{a_{i}}
$$

Cuando se tienen varias plantas, los términos $P_{G i}$ se suman y se expresan como un $P_{\text {GTotal }}$, obteniendo la ecuación (14).

$$
P_{\text {GTotal }}=\frac{\lambda_{1}-b_{1}}{a_{1}}+\frac{\lambda_{2}-b_{2}}{a_{2}}+\ldots \ldots+\frac{\lambda_{i}-b_{i}}{a_{i}}
$$


Si se tienen $i$ generadores conectados, el criterio de punto óptimo para el OPF clásico sin restricciones, se obtiene cuando se cumple la condición descrita en la ecuación (15).

$$
\lambda_{\text {óptimo }}=\lambda_{1}=\lambda_{2}=\ldots=\lambda_{i}
$$

Por lo tanto, de acuerdo al criterio de optimización, la ecuación (14) se puede expresar a través de la ecuación (16)

$$
P_{\text {GTotal }}=\frac{\lambda_{\text {óptimo }}-b_{1}}{a_{1}}+\frac{\lambda_{\text {óptimo }}-b_{2}}{a_{2}}+\ldots \ldots+\frac{\lambda_{\text {óptimo }}-b_{i}}{a_{i}}
$$

Despejando $\lambda_{\text {ótimo }}$ de la ecuación (16) y generalizando los términos $a_{i}, b_{i}$ y $P_{\text {GTotal }}$ como sumatorias para $i$ generadores se obtiene la ecuación (17).

$$
\lambda_{\text {optimo }}=\left(\sum_{n=1}^{i} \frac{1}{a_{n}}\right)^{-1}\left(P_{\text {GTotal }}\right)+\left(\sum_{n=1}^{i} \frac{1}{a_{n}}\right)^{-1}\left(\sum_{n=1}^{i} \frac{b_{n}}{a_{n}}\right)
$$

\subsubsection{Despacho con pérdidas de potencia activa}

Es un despacho teniendo en cuenta las pérdidas de la red de transmisión $\left(P_{L} \neq 0\right)$ y sin límites de generación. Incluir las pérdidas produce que el costo marginal de abastecer la demanda local no sea único a través de la red de transmisión, esto indica que no se cumple el criterio descrito en la ecuación (15). Para el análisis del OPF con perdidas, los multiplicadores de Lagrange permiten encontrar el despacho de las máquinas de generación (ecuación (18)), minimizando los costos de la función descrita en la ecuación (4), sujeta a la restricción del balance de potencia (ecuación (2)).

$$
l(P, \lambda)=\left(\sum_{n=1}^{i} C_{n}\left(P_{G n}\right)\right)+\lambda\left(\sum P_{D}+P_{L}\left(P_{G}, P_{D}\right)-\sum_{n=1}^{i} P_{G n}\right)
$$

De acuerdo a la condición de primer orden, se obtiene la ecuación (19) y la ecuación (20). Donde la ecuación (19) permite determinar la potencia que debe generar cada máquina conectada al sistema y la ecuación (20) representa el balance de potencia, parámetro que posteriormente será la condición de parada del método numérico a implementar.

$$
\begin{gathered}
\nabla_{P i} l(P, \lambda)=\frac{\partial C_{i}}{\partial P_{G i}}+\lambda\left(\frac{\partial P_{L}}{\partial P_{G i}}-1\right) \\
\nabla_{\lambda} l(P, \lambda)=\sum P_{D}+P_{L}\left(P_{G}, P_{D}\right)-\sum_{n=1}^{i} P_{G n}
\end{gathered}
$$

2.3.3. Condiciones de primer orden $\nabla_{P i} l(P, \lambda)$

La condición de primer orden (ecuación (19)) establece la potencia activa $P_{G i}$ que debe despachar cada máquina, esta condición incluye el factor de penalización de pérdidas $(\varphi)$, factor que se presenta en la ecuación (21).

$$
\varphi=\frac{\partial P_{L}}{\partial P_{G i}}-1
$$


Para determinar el despacho se requiere establecer el factor de penalización, criterio que depende de la función de pérdidas $P_{L}$, la cual se puede expresar como un conjunto de sumatorias, como se muestra en la ecuación (22). Donde $B$ representa la parte real de la matriz Hermitiana.

$$
P_{L}=\sum_{r=1}^{i} \sum_{s=1}^{i} P_{G r} B_{r s} P_{G s}+\sum_{r=1}^{i} B_{r 0} P_{G r}+B_{00}
$$

De acuerdo a la función de costos (ecuación (4)), al criterio de primer orden (ecuación (19)) y a la función de pérdidas (ecuación (22)), la potencia $P_{G i}$ que debe despachar cada máquina se puede obtener a través de la ecuación (23).

$$
\left[\begin{array}{c}
P_{G 1} \\
P_{G 2} \\
\vdots \\
P_{G i}
\end{array}\right]=\left[\begin{array}{cccc}
\left(\frac{a_{1}}{\lambda_{\text {optimo }}}+2 B_{11}\right) & 2 B_{12} & \cdots & 2 B_{1 i} \\
2 B_{21} & \left(\frac{a_{2}}{\lambda_{\text {optimo }}}+2 B_{22}\right) & \cdots & 2 B_{2 i} \\
\vdots & \vdots & \ddots & \vdots \\
2 B_{i 1} & 2 B_{i 2} & \cdots & \left(\frac{a_{i}}{\lambda_{\text {optimo }}}+2 B_{i i}\right)
\end{array}\right]^{-1} \cdot\left[\begin{array}{c}
\left(1-B_{10}\right)-\frac{b_{1}}{\lambda_{\text {optimo }}} \\
\left(1-B_{20}\right)-\frac{b_{2}}{\lambda_{\text {optimo }}} \\
\vdots \\
\left(1-B_{i 0}\right)-\frac{b_{i}}{\lambda_{\text {optimo }}}
\end{array}\right]
$$

\section{Metodología}

La contribución de este trabajo es el desarrollo de una herramienta de simulación que permita analizar el flujo óptimo de sistemas de potencia, minimizando los costos de generación para el despacho de plantas térmicas usando el método de los multiplicadores de Lagrange. El simulador se denomina SOPF (Software Optimal Power Flow), fue desarrollado en Guide-Matlab R2018b. El simulador se diseño en una estructura por módulos. A continuación se presenta la descripción de los módulos y los algoritmos de cada uno de los módulos.

\subsection{Módulos principales}

Los módulos principales, como los muestra la figura (2) contienen la estructura de la aplicación asociada a la restricción de las pérdidas de potencia activa, no se contemplan límites de operación.

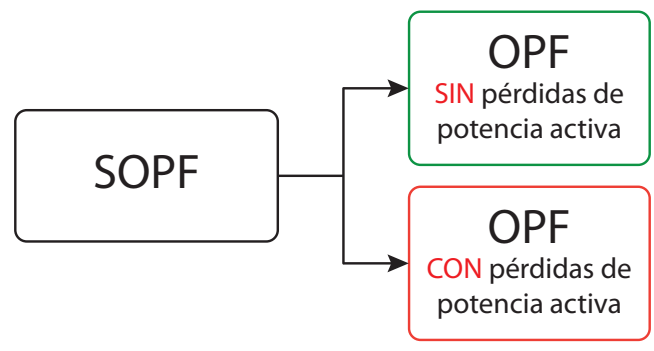

Figure 2. Estructura de SOPF

El primer módulo: OPF sin pérdidas de potencia activa permite realizar el OPF de acuerdo al criterio de punto de operación óptimo, donde se establece que un despacho de operación térmica es óptimo para $i$ centrales sino se produce ninguna ganancia adicional por transferencia de un generador a otro, el problema de optimización no está acotado y la solución se realiza a través del criterio de costo incremental. El Algoritmo 1 presenta el pseudocódigo de este módulo principal.

El segundo módulo: OPF con pérdidas de potencia activa realiza el OPF incluyendo la restricción de igualdad asociada a las pérdidas de potencia activa del sistema. Se utiliza la formulación descrita en la introducción. El Algoritmo 2 presenta el pseudocódigo de este módulo principal. 


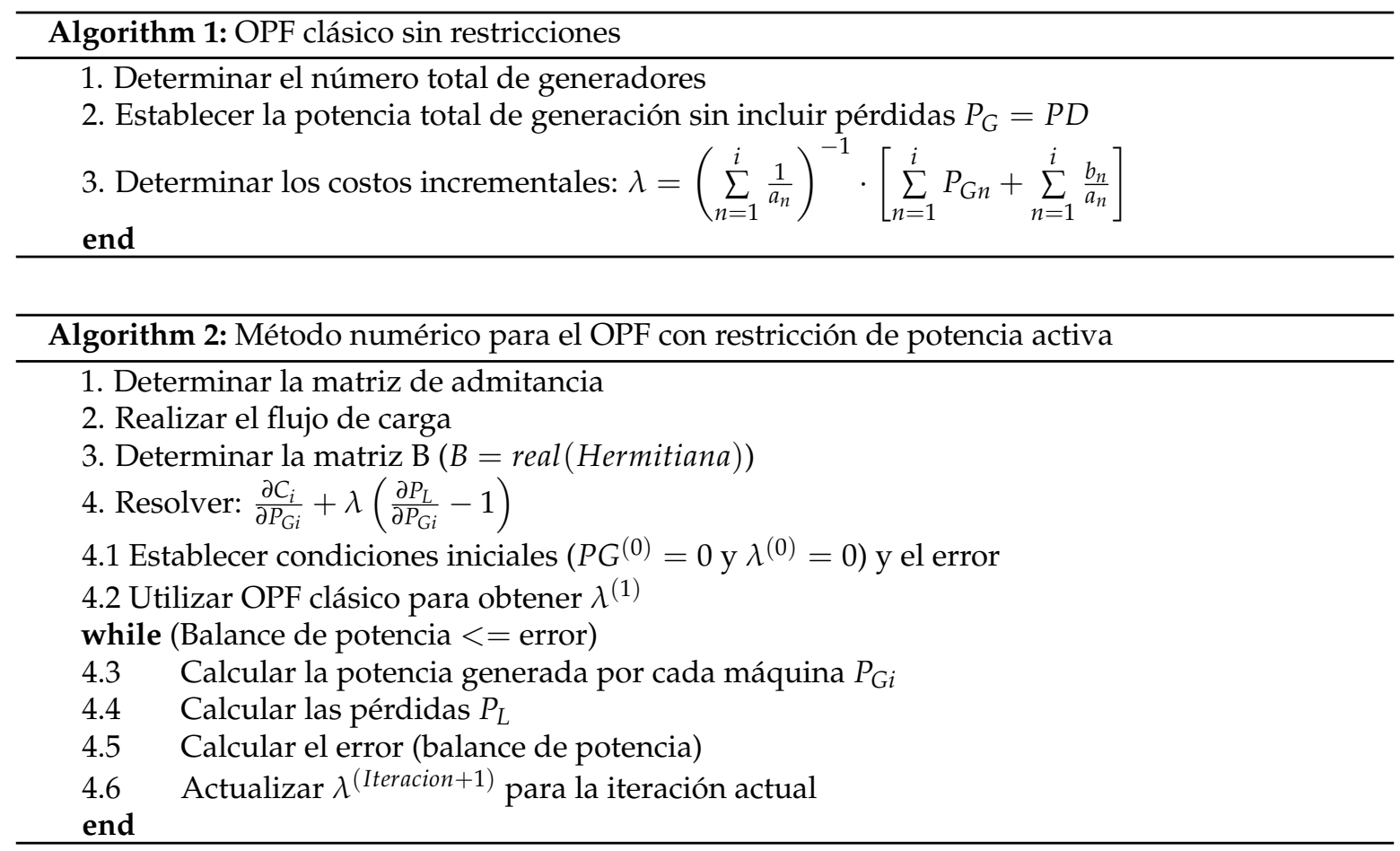

\subsection{Módulos auxiliares}

Los módulos auxiliares son variantes de los módulos principales, contienen la estructura de programación asociada a las restricciones de operación. Estos módulos permiten acotar el problema de optimización de acuerdo a la máxima y mínima potencia que puede generar una central térmica.

La Figura (3) presenta el diagrama de bloques, con el control de variables de entradas y salidas para el módulo principal OPF sin pérdidas de potencia activa incluyendo el módulo auxiliar de restricciones de operación.

La Figura (4) presenta el diagrama de bloques, con el control de variables de entradas y salidas para el módulo principal OPF con pérdidas de potencia activa incluyendo el módulo auxiliar de restricciones de operación.

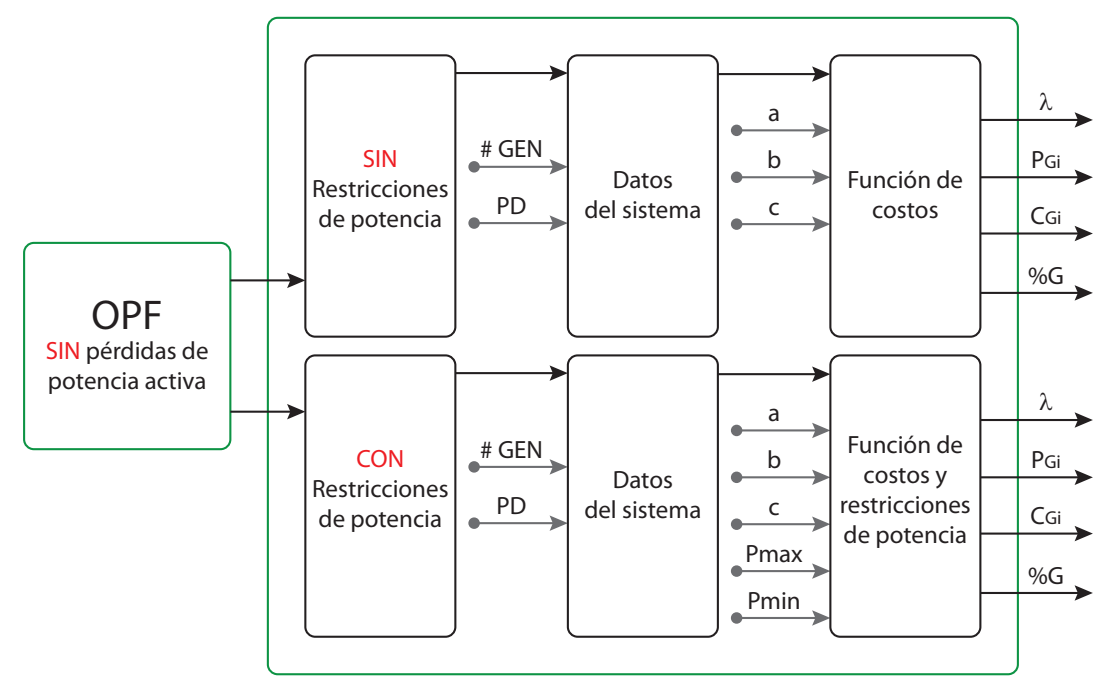

Figure 3. Diagrama de bloques OPF sin pérdidas de potencia activa 


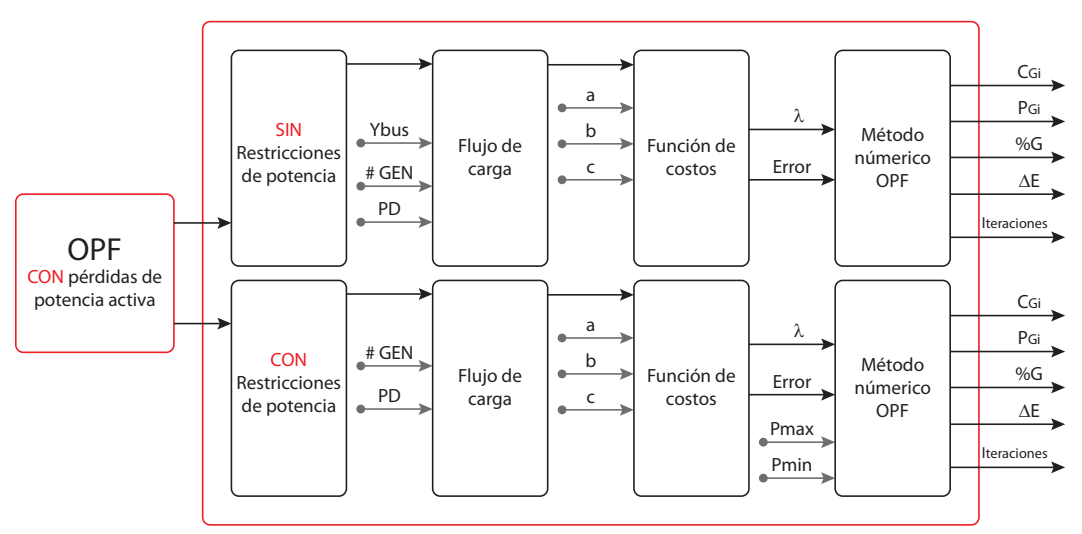

Figure 4. Diagrama de bloques OPF con pérdidas de potencia activa

El Algoritmo 3 presenta el pseudocódigo de los módulos auxiliares, es una estructura común que se aplica de la misma forma para el módulo OPF sin pérdidas de potencia activa y para el módulo OPF con pérdidas de potencia activa. Como se muestra en el Algoritmo 3, el criterio presentado se desarrolla a un solo generador.

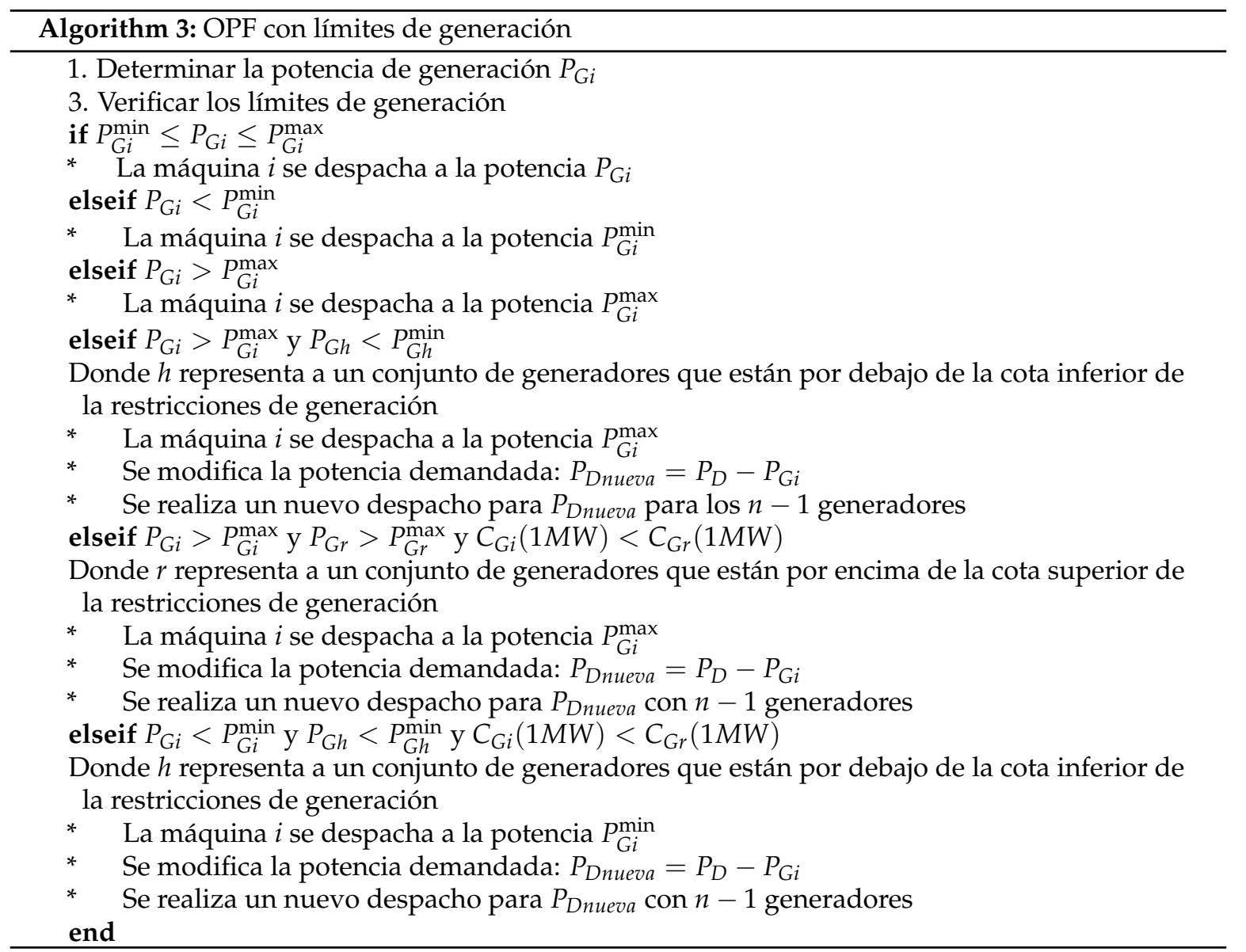

\subsection{Herramienta de simulación}

El simulador está disponible para la comunidad académica a través del siguiente enlace: http: / / repository.udistrital.edu.co/handle/11349/16140. Se puede descargar de forma gratuita, solo se requiere tener instalado Matlab Runtime Installer. Adicionalmente, el simulador cuenta con un manual 
para SOPF y unas guías teóricas detalladas sobre OPF. En la figura (5) y figura (6) se presenta la interfaz del simulador de acuerdo al estudio de caso a analizar.

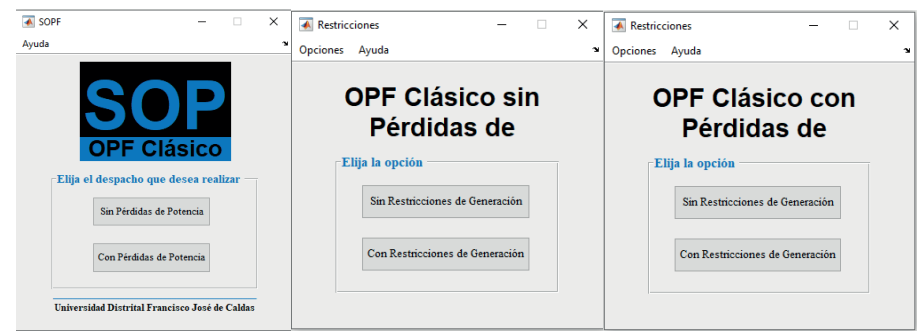

Figure 5. Interfaz principales SOPF

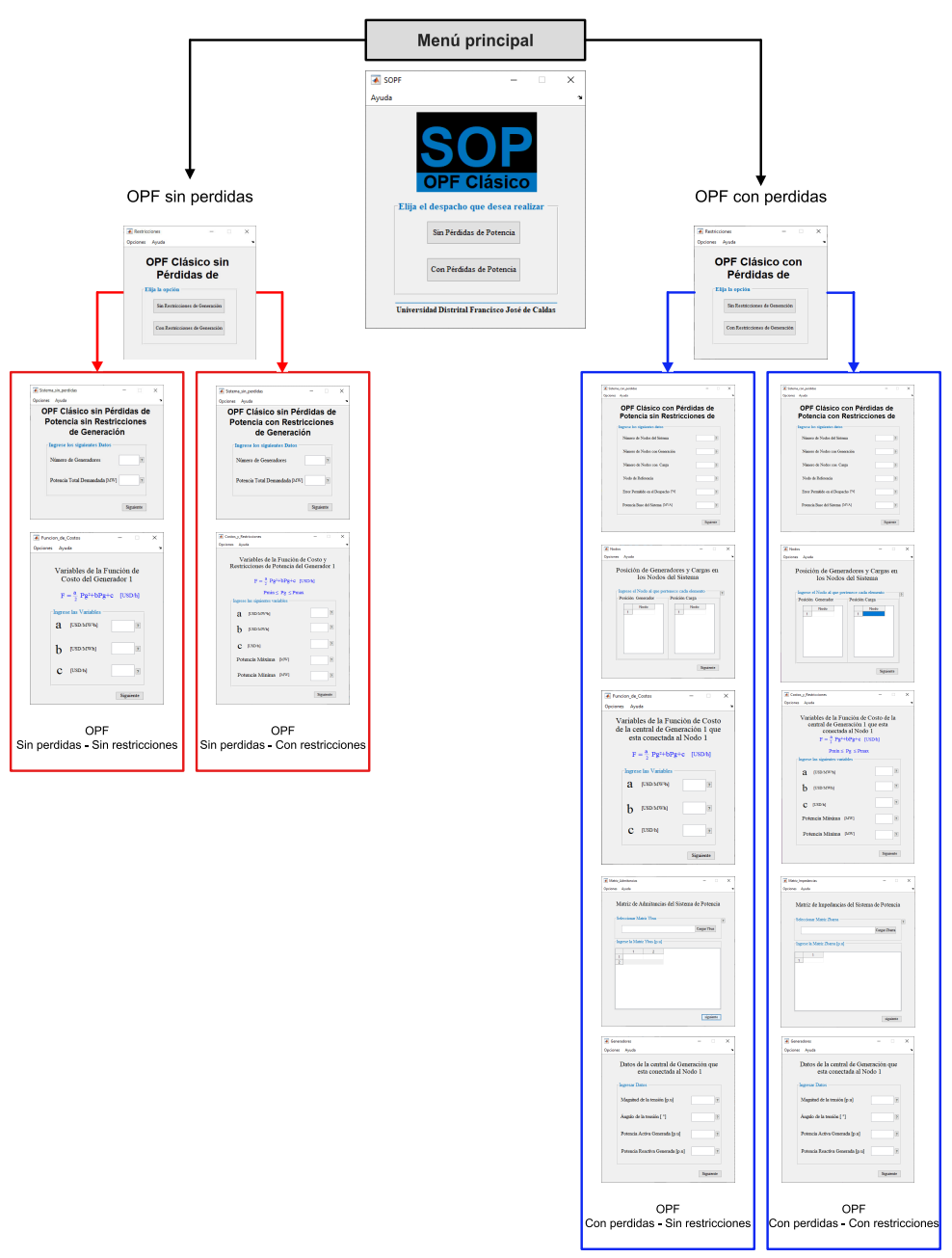

Figure 6. Ventanas SOPF de acuerdo al caso de estudio

\section{Resultados}

Para evaluar la eficiencia del software desarrollado, se proponen dos casos de estudio, esta selección se realiza teniendo en cuenta los escenarios donde se presenta mayor carga computacional. El primer caso es un despacho con pérdidas y sin restricciones de potencia activa y el segundo caso es un despacho con pérdidas y con límites de generación. No se implementa el despacho sin pérdidas, debido a que analíticamente, es sencillo encontrar el criterio de punto óptimo. 
Como análisis comparativo, se determina el error en función de la solución teórica del método. A continuación, se presentan los casos de estudio implementados y el respectivo cálculo de error tomando como valores reales los resultados teóricos. La descripción detallada de los cálculos teóricos se presenta en (Castro Rico et al. 2019).

La implementación se realizó en un equipo con procesador Intel (R) Core (TM) i7-7700HQ de 2.8 GHz con 24 GB de RAM, sistema operativo Microsoft Windows 10 de 64 bits usando Matlab versión R2018b.

\subsection{Caso de estudio 1: Flujo óptimo clásico con pérdidas y sin restricciones de potencia activa}

Para el análisis del OPF con pérdidas y sin restricciones de potencia activa se implementa el sistema de potencia de cuatro nodos de la Figura (7). El objetivo es determinar la potencia generada de $\mathrm{Gen}_{1}$ y $\mathrm{Gen}_{2}$ con un error de $1 \%$ utilizando una potencia base de $100 \mathrm{MVA}$.

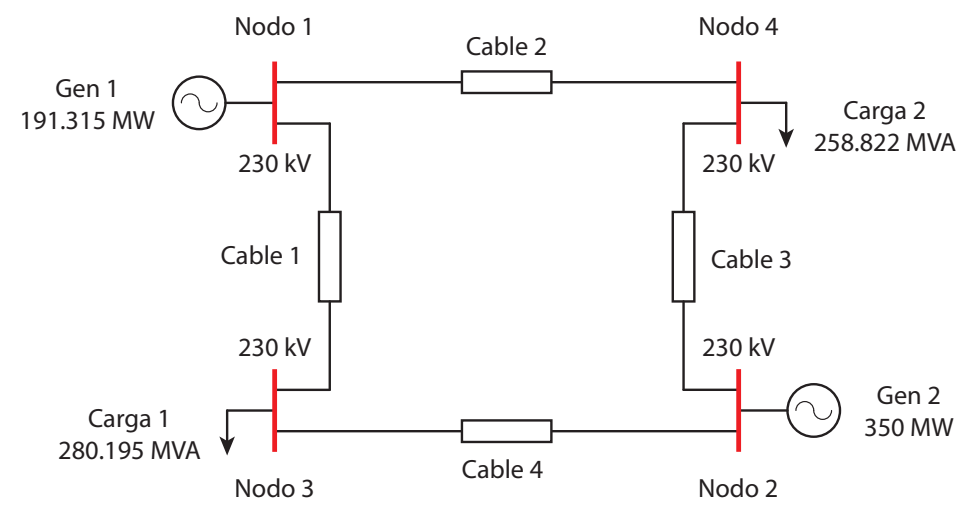

Figure 7. Sistemas de potencia cuatro nodos caso de estudio 1

\subsubsection{Función de costos}

Las funciones de costos asociadas a las plantas de generación se presentan en las ecuaciones (24) y (25).

$$
\begin{array}{cc}
C_{1}\left(P_{G e n 1}\right)=\frac{0.008}{2}\left(P_{G e n 1}\right)^{2}+8\left(P_{G e n 1}\right)+180 & {\left[\frac{\$}{h}\right]} \\
C_{2}\left(P_{G e n 2}\right)=\frac{0.0096}{2}\left(P_{G e n 2}\right)^{2}+6.4\left(P_{G e n 2}\right)+250 & {\left[\begin{array}{l}
\$ \\
h
\end{array}\right]}
\end{array}
$$

\subsubsection{Parámetros del flujo de carga}

En la tabla (1) se presentan los modelos de las líneas, las tensiones y la potencia demandada y generada por nodo (todos los datos están en por unidad). El modelo del sistema de potencia se presenta a través de la matriz de admitancia de la ecuación (26).

Table 1. Características del sistema para el caso de estudio 1

\begin{tabular}{|c|c|c|c|c|c|c|c|c|c|}
\hline \multicolumn{4}{|c|}{ Datos Líneas } & \multicolumn{6}{c|}{ Datos Nodo } \\
\hline \multirow{2}{*}{ Nodo } & \multicolumn{2}{|c|}{$Z_{\text {serie }}$} & $Y_{\text {Paralelo }}$ & \multirow{2}{*}{ Nodo } & \multicolumn{2}{c|}{ Demanda } & Generación & \multirow{2}{*}{$|V|$} \\
\cline { 2 - 6 } \cline { 6 - 8 } & $\mathrm{R}$ & $\mathrm{X}$ & $\mathrm{B}$ & & $\mathrm{P}$ & $\mathrm{Q}$ & $\mathrm{P}$ & $\mathrm{Q}$ & \\
\hline $1-4$ & 0.00744 & 0.0372 & 0.0775 & 1 & 0 & 0 & - & - & 1.0 \\
\hline $1-3$ & 0.01008 & 0.0504 & 0.1025 & 2 & 0 & 0 & 3.18 & - & 1.0 \\
\hline $2-3$ & 0.00744 & 0.0372 & 0.0775 & 3 & 2.20 & 1.3634 & 0 & 0 & - \\
\hline $2-4$ & 0.01272 & 0.0636 & 0.1275 & 4 & 2.80 & 1.7352 & 0 & 0 & - \\
\hline
\end{tabular}




$$
\mathbf{Y}_{\text {bus }}=\left[\begin{array}{cccc}
8.9854-j 44.836 & -0.0004+j 0.0010 & -3.8154+j 19.077 & -5.1696+j 25.848 \\
-0.0004+j 0.0010 & 8.1934-j 40.864 & -5.1696+j 25.848 & -3.0233+j 15.117 \\
-3.8154+j 19.077 & -5.1696+j 25.848 & 8.9854-j 44.836 & -0.0004+j 0.0010 \\
-5.1696+j 25.848 & -3.0233+j 15.117 & -0.0004+j 0.0010 & 8.1934-j 40.864
\end{array}\right]
$$

El flujo de carga en por unidad de acuerdo a la matriz de admitancia y a la información de la tabla (1) se describe en la tabla (2).

Table 2. Flujo de carga caso de estudio 1

\begin{tabular}{|c|c|c|c|c|c|c|}
\hline \multirow{2}{*}{ Nodo } & \multicolumn{2}{|c|}{ Demanda } & \multicolumn{2}{c|}{ Generación } & \multicolumn{2}{c|}{ Tensión } \\
\cline { 2 - 7 } & $\mathrm{P}$ & $\mathrm{Q}$ & $\mathrm{P}$ & $\mathrm{Q}$ & Magnitud & Ángulo \\
\hline 1 & 0 & 0 & 1.913152 & 1.872240 & 1 & 0 \\
\hline 2 & 0 & 0 & 3.18 & 1.325439 & 1 & 2.43995 \\
\hline 3 & 2.20 & 1.3634 & 0 & 0 & 0.96051 & -1.07932 \\
\hline 4 & 2.80 & 1.7352 & 0 & 0 & 0.94304 & -2.62658 \\
\hline
\end{tabular}

\subsubsection{Flujo de carga óptimo}

En la tabla (3) se presentan los resultados teóricos y en la tabla (4) los obtenidos a través del programa SOPF. Para cumplir con el error definido fue necesario de cuatro iteraciones. Todos los datos se presentan en valores en por unidad.

Table 3. Resultados teóricos OPF caso de estudio 1 (Castro Rico et al. 2019)

\begin{tabular}{|c|c|c|c|c|c|}
\hline & \multicolumn{5}{|c|}{ Valor Teórico } \\
\cline { 2 - 6 } & $\lambda$ & $P g_{1}$ & $P g_{2}$ & $P_{L}$ & $\varepsilon$ \\
\hline Iteración 1 & 9.4545 & 1.5128 & 2.8452 & 0.0693 & 0.7112 \\
\hline Iteración 2 & 10.9975 & 3.0413 & 4.2125 & 0.1861 & -2.0678 \\
\hline Iteración 3 & 9.8957 & 1.9582 & 3.2409 & 0.0969 & -0.1021 \\
\hline Iteración 4 & 9.8409 & 1.9032 & 3.1919 & 0.0932 & -0.0019 \\
\hline
\end{tabular}

Table 4. Resultados obtenidos a través de SOPF para el caso de estudio 1

\begin{tabular}{|c|c|c|c|c|c|}
\hline & \multicolumn{5}{|c|}{ Valor Matlab } \\
\cline { 2 - 6 } & $\lambda$ & $P g_{1}$ & $P g_{2}$ & $P_{L}$ & $\varepsilon$ \\
\hline Iteración 1 & 9.4545 & 1.5128 & 2.8453 & 0.0682 & 0.7100 \\
\hline Iteración 2 & 10.9948 & 3.0388 & 4.2102 & 0.1835 & -2.0655 \\
\hline Iteración 3 & 9.8943 & 1.9567 & 3.2397 & 0.0953 & -0.1011 \\
\hline Iteración 4 & 9.8400 & 1.9023 & 3.1913 & 0.0917 & -0.0019 \\
\hline
\end{tabular}

Para establecer la exactitud de los resultados obtenidos a través de SOPF se procede a determinar el error relativo, se utiliza como valor real el despacho obtenido a través de forma teórica. El error para cada una de las iteraciones se presenta en la tabla (5), se obtiene un error menor al $1 \%$

Table 5. Error relativo entre OPF teórico y SOPF para el caso de estudio 1

\begin{tabular}{|c|c|c|c|c|}
\hline & \multicolumn{4}{|c|}{ Error Relativo } \\
\cline { 2 - 5 } & $\lambda$ & $P g_{1}$ & $P g_{2}$ & $P_{L}$ \\
\hline Iteración 1 & 0 & 0 & 0 & 0.0158 \\
\hline Iteración 2 & 0.0002 & 0.0008 & 0.0005 & 0.0139 \\
\hline Iteración 3 & -0.0001 & 0.0007 & 0.0003 & 0.0165 \\
\hline Iteración 4 & 0 & 0.0004 & 0.00001 & 0.0160 \\
\hline
\end{tabular}




\subsection{Caso de estudio 2: Flujo óptimo clásico con pérdidas y con limites de generación}

Para el análisis del OPF con pérdidas y con restricciones de potencia activa se implementa el sistema de potencia de cuatro nodos de la Figura (8). El objetivo es determinar la potencia generada de $\mathrm{Gen}_{1}$ y Gen 2 con un error de 1 \% utilizando una potencia base de $100 \mathrm{MVA}$.

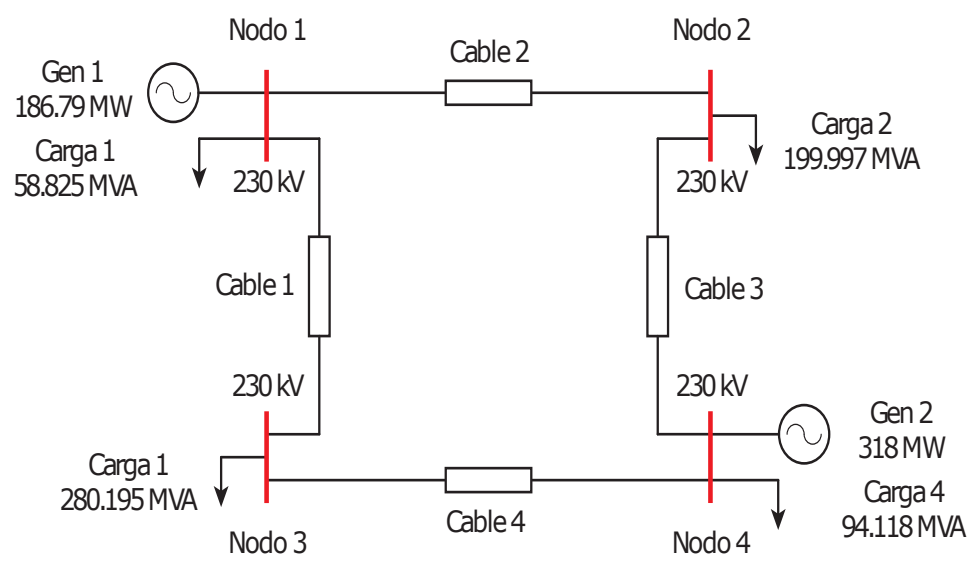

Figure 8. Sistemas de potencia cuatro nodos caso de estudio 2

\subsubsection{Función de costos}

Las funciones de costos asociadas a las plantas de generación se presentan en las ecuaciones (27) y (28).

$$
\begin{array}{ll}
C_{1}\left(P_{\text {Gen } 1}\right)=\frac{0.074}{2}\left(P_{G e n 1}\right)^{2}+6.6\left(P_{G e n 1}\right)+175 & {\left[\frac{U S D}{h}\right]} \\
C_{1}\left(P_{G e n 2}\right)=\frac{0.009}{2}\left(P_{G e n 2}\right)^{2}+8.1\left(P_{G e n 2}\right)+210 & {\left[\frac{U S D}{h}\right]}
\end{array}
$$

Las restricciones de potencia activa para cada una de las máquinas se presenta en las ecuaciones (29) y (30).

$$
\begin{gathered}
80[\mathrm{MW}] \leq P_{\mathrm{Gen} 1} \leq 220[\mathrm{MW}] \\
130[\mathrm{MW}] \leq P_{\mathrm{Gen} 2} \leq 300[\mathrm{MW}]
\end{gathered}
$$

\subsubsection{Parámetros del flujo de carga}

En la tabla (6) se presentan los modelos de las líneas, las tensiones y la potencia demandada y generada por nodo (todos los datos están en por unidad). El modelo del sistema de potencia se presenta a través de la matriz de admitancia de la ecuación (31).

Table 6. Características del sistema para el caso de estudio 2 (Castro Rico et al. 2019)

\begin{tabular}{|c|c|c|c|c|c|c|c|c|c|}
\hline \multicolumn{4}{|c|}{ Datos Líneas } & \multicolumn{6}{c|}{ Datos Nodo } \\
\hline \multirow{2}{*}{ Nodo } & \multicolumn{2}{|c|}{$Z_{\text {serie }}$} & $Y_{\text {Paralelo }}$ & \multirow{2}{*}{ Nodo } & \multicolumn{2}{|c|}{ Demanda } & Generación & \multirow{2}{*}{$|V|$} \\
\cline { 2 - 6 } & $\mathrm{R}$ & $\mathrm{X}$ & $\mathrm{B}$ & & $\mathrm{P}$ & $\mathrm{Q}$ & $\mathrm{P}$ & $\mathrm{Q}$ & \\
\hline $1-4$ & 0.00744 & 0.0372 & 0.0775 & 1 & 0.50 & 0.31 & - & - & 1.0 \\
\hline $1-3$ & 0.01008 & 0.0504 & 0.1025 & 2 & 1.70 & 1.054 & 0 & 0 & - \\
\hline $2-3$ & 0.00744 & 0.0372 & 0.0775 & 3 & 2.0 & 1.239 & 0 & 0 & - \\
\hline $2-4$ & 0.01272 & 0.0636 & 0.1275 & 4 & 0.80 & 0.50 & 3.18 & - & 1.02 \\
\hline
\end{tabular}




$$
\mathbf{Y}_{\text {bus }}=\left[\begin{array}{cccc}
8.9852-j 448360 & -3.8156+j 19.0781 & -5.1696+j 25.8478 & 0 \\
-3.8156+j 19.0781 & 8.9852-j 44.8360 & 0 & -5.1696+j 25.8478 \\
-5.1696+j 25.8478 & 0 & 8.9852-j 44.8360 & -3.0237+j 15.1185 \\
0 & -5.1696+j 25.8478 & -3.0237+j 15.1185 & 8.1933-j 40.8638
\end{array}\right]
$$

El flujo de carga en por unidad de acuerdo a la matriz de admitancia y a la información de la tabla (6) se describe en la tabla (7).

Table 7. Flujo de carga caso de estudio 2

\begin{tabular}{|c|c|c|c|c|c|c|}
\hline \multirow{2}{*}{ Nodo } & \multicolumn{2}{|c|}{ Demanda } & \multicolumn{2}{c|}{ Generación } & \multicolumn{2}{c|}{ Tensión } \\
\cline { 2 - 7 } & $\mathrm{P}$ & $\mathrm{Q}$ & $\mathrm{P}$ & $\mathrm{Q}$ & Magnitud & Ángulo \\
\hline 1 & 0.50 & 0.31 & 1.87 & 1.15 & 1 & 0 \\
\hline 2 & 1.70 & 1.054 & 0 & 0 & 0.98 & -0.98 \\
\hline 3 & 2.0 & 1.239 & 0 & 0 & 0.97 & -1.87 \\
\hline 4 & 0.80 & 0.50 & 3.18 & 1.82 & 1.02 & 1.53 \\
\hline
\end{tabular}

\subsubsection{Flujo de carga óptimo}

En la tabla (8) se presentan los resultados teóricos y en la tabla (9) los obtenidos a través del programa SOPF. Para cumplir con el error definido fue necesario de siete iteraciones. Todos los datos se presentan en valores en por unidad.

Table 8. Resultados teóricos OPF caso de estudio 2

\begin{tabular}{|c|c|c|c|c|c|}
\hline & \multicolumn{5}{|c|}{ Valores Teóricos } \\
\cline { 2 - 6 } & $\lambda$ & $P g_{1}$ & $P_{2}$ & $P_{L}$ & $\varepsilon$ \\
\hline Iteración 1 & 11.9493 & 0.7285 & 3.8321 & 0.0633 & 0.5027 \\
\hline Iteración 2 & 13.2665 & 0.9103 & 5.0837 & 0.1114 & -0.8826 \\
\hline Iteración 3 & 12.4555 & 0.7983 & 4.3164 & 0.0803 & -0.0344 \\
\hline Iteración 4 & 12.4229 & 0.7939 & 4.2862 & 0.0792 & -0.00089 \\
\hline Iteración 5 & 21.4 & 1.9433 & 3.0 & 0.0447 & 0.1014 \\
\hline Iteración 6 & 14.7482 & 1.0792 & 3.0 & 0.0389 & 0.9597 \\
\hline Iteración 7 & 22.1357 & 2.0380 & 3.0 & 0.0458 & 0.0078 \\
\hline
\end{tabular}

Table 9. Resultados obtenidos a través de SOPF para el caso de estudio 2

\begin{tabular}{|c|c|c|c|c|c|}
\hline & \multicolumn{5}{|c|}{ Valor Matlab } \\
\cline { 2 - 6 } & $\lambda$ & $P g_{1}$ & $P_{2}$ & $P_{L}$ & $\varepsilon$ \\
\hline Iteración 1 & 11.9493 & 0.7285 & 3.8321 & 0.0631 & 0.5025 \\
\hline Iteración 2 & 13.2662 & 0,9102 & 5.0832 & 0.1112 & -0.8823 \\
\hline Iteración 3 & 12.4554 & 0.7982 & 4.3162 & 0.0801 & -0.0343 \\
\hline Iteración 4 & 12.4236 & 0.7939 & 4.2860 & 0.0790 & -0.0009 \\
\hline Iteración 5 & 21.4 & 1.9439 & 3.0 & 0.0442 & -0.1009 \\
\hline Iteración 6 & 14.7676 & 1.0817 & 3.0 & 0.0386 & -0.9568 \\
\hline Iteración 7 & 22.1340 & 2.0377 & 3.0 & 0.0453 & -0.0076 \\
\hline
\end{tabular}

Para establecer la exactitud de los resultados obtenidos a través de SOPF se procede a determinar el error relativo, se utiliza como valor real el despacho obtenido a través de forma teórica. El error para cada una de las iteraciones se presenta en la tabla (10). Como se puede observar el mayor error es de $0.2571 \%$, lo que indica que el error de SOPF no supera el $1 \%$. De acuerdo al análisis fue necesario despachar la planta 2 a su máxima potencia de generación debido a que supero su restricción de generación. 
Table 10. Error relativo entre OPF teórico y SOPF para el caso de estudio 2

\begin{tabular}{|c|c|c|c|c|}
\hline & \multicolumn{4}{|c|}{ Error Relativo } \\
\cline { 2 - 5 } & $\lambda$ & $P g_{1}$ & $P_{2}$ & $P_{L}$ \\
\hline Iteración 1 & 0 & 0 & 0.0007 & 0.0017 \\
\hline Iteración 2 & 0.0002 & 0 & 0 & 0.0017 \\
\hline Iteración 3 & 0.0001 & 0 & 0 & 0.0015 \\
\hline Iteración 4 & 0 & 0 & 0 & 0.0018 \\
\hline Iteración 5 & 0 & 0 & 0 & 0.0094 \\
\hline Iteración 6 & 0.0010 & 0.0023 & 0 & 0.0060 \\
\hline Iteración 7 & 0 & 0.0010 & 0 & 0.0099 \\
\hline
\end{tabular}

\section{Conclusiones y Discusión}

Se desarrolla una aplicación de acceso libre que minimiza los costos de generación para el despacho térmico utilizando como estrategia de solución los multiplicadores de Lagrange. La herramienta denominada SOPF permite parametrizar los elementos de un sistema de potencia para realizar flujo óptimo teniendo en cuenta pérdidas de potencia activa. SOPF determina los porcentajes de participación de cada planta, de acuerdo a los límites de generación asociados a las restricciones de operación. El simulador tiene la capacidad de generar un historial con los resultados de las iteraciones hasta cumplir con los parámetros del sistema de potencia ingresado. Como métricas, el simulador determina: el despacho de la potencia activa de cada generador, los costos de generación de la potencia despachada y el aporte de cada máquina en términos porcentuales. SOPF brinda la posibilidad de ser un apoyo para los estudiantes, profesores y personas interesadas en la aplicación de algoritmos de optimización para la operación económica de sistemas eléctricos de potencia.

Los resultados se presentan a través de dos casos de estudio: flujo óptimo clásico con pérdidas y sin restricciones de potencia activa y flujo óptimo clásico con pérdidas y con restricciones de potencia activa. Para ambos casos, se obtienen errores inferiores al $1 \%$.

Contribución de los autores: Conceptualización, D. A., J. C and D. G.; Metodología, D. A and J. C; Investigación, D. A., J. C and D. G.; Escritura, revisión y edición, D. G.

Financiamiento y Agradecimientos: Esta investigación fue financiada por la Universidad Distrital Francisco José de Caldas - Facultad Tecnológica. Los autores agradecen a la Universidad Distrital Francisco José de Caldas.

Conflicto de intereses: Los autores declaran no tener ningún conflicto de intereses.

\section{References}

Alvarez-Bustos, A., Kazemtabrizi, B., Shahbazi, M., and Acha-Daza, E. (2021). Universal branch model for the solution of optimal power flows in hybrid AC/DC grids. International Journal of Electrical Power E Energy Systems, 126:106543. doi:https:/ /doi.org/10.1016/j.ijepes.2020.106543.

Bukhsh, W., Edmunds, C., and Bell, K. (2020). OATS: Optimisation and Analysis Toolbox for Power Systems. IEEE Transactions on Power Systems, 35(5):3552-3561. doi:10.1109/TPWRS.2020.2986081.

Castro Rico, J. E., Anzola Bustos, D. A., and Giral, D. (2019). Software en Matlab para el flujo óptimo clásico para el despacho hidrotérmico. PhD thesis, Universidad Distrital Francisco José de Caldas.

Chaudhary, R., Sethi, S., Keshari, R., and Goel, S. (2012). A study of comparison of Network Simulator -3 and Network Simulator -2. International Journal of Computer Science and Information Technologies.

Chen, H., Ngan, H., and Zhang, Y. (2016). Power system optimization: large-scale complex systems approaches. John Wiley \& Sons.

Cuffe, P. (2020). Optimization and Visualization Tools for Situational Awareness in Highly Renewable Power Systems. In 2020 6th IEEE International Energy Conference (ENERGYCon), pages 930-933. doi:10.1109/ENERGYCon48941.2020.9236524. 
Das, J. C. (2017). Load flow optimization and optimal power flow. Crc Press.

Grisales-Noreña, L. F., Garzón-Rivera, O. D., Ocampo-Toro, J. A., Ramos-Paja, C. A., and Rodriguez-Cabal, M. A. (2020). Metaheuristic Optimization Methods for Optimal Power Flow Analysis in DC Distribution Networks. Transactions on Energy Systems and Engineering Applications, 1(1):13-31. doi:10.32397/tesea.vol1.n1.2.

Hasan, F., Kargarian, A., and Mohammadi, A. (2020). A survey on applications of machine learning for optimal power flow. In 2020 IEEE Texas Power and Energy Conference, TPEC 2020. doi:10.1109/TPEC48276.2020.9042547. Ilyas, A. M., Suyuti, A., Gunadin, I. C., and Siswanto, A. (2020). Optimal Power Flow the Sulselrabar 150 KV system before and after the penetration of wind power plants considering power loss and generation costs. IOP Conference Series: Materials Science and Engineering, 850:12030. doi:10.1088/1757-899x/850/1/012030.

Ma, X. and Elia, N. (2019). Optimization Dynamics: A Bus-Level Distributed Approach for Optimal Power Flows. IEEE Transactions on Control of Network Systems, 6(2):642-652. doi:10.1109/TCNS.2018.2864140.

Montoya, O. D. (2017). Solving a Classical Optimization Problem Using GAMS Optimizer Package: Economic Dispatch Problem Implementation. Ingeniería y Ciencia, 13(26):39-63. doi:10.17230/ingciencia.13.26.2.

Pinheiro, R. B. N. M., Nepomuceno, L., and Balbo, A. R. (2020). Solving large-scale reactive optimal power flow problems by a primal-dual M2BF approach. Optimization and Engineering, 21(2):485-515. doi:10.1007/s11081-019-09451-4.

Thurner, L., Scheidler, A., Schäfer, F., Menke, J., Dollichon, J., Meier, F., Meinecke, S., and Braun, M. (2018). Pandapower-An Open-Source Python Tool for Convenient Modeling, Analysis, and Optimization of Electric Power Systems. IEEE Transactions on Power Systems, 33(6):6510-6521. doi:10.1109/TPWRS.2018.2829021.

Wood, A. J., Wollenberg, B. F., and Sheblé, G. B. (2013). Power generation, operation, and control. John Wiley \& Sons.

(C) 2021 by the authors. Licensee TESEA, Cartagena, Colombia. This article is an open access article distributed under the terms and conditions of the Creative Commons Attribution (CC BY) license (http:/ / creativecommons.org/licenses/by/4.0/). 